1 from each side, and crossed in the midline, creating a sphincter over the tube. The incorporation of this continence procedure enables the surgeon to do minimal mobilization of the anterior wall of the bladder, almost like performing an open incontinent cystostomy. One of the most appealing indications for using this approach is the prune-belly syndrome. ${ }^{1}$

Respectfully,

Antonio Macedo, Jr.

Department of Urology Federal University of São Paulo São Paulo, Brazil

1. Macedo A Jr, Rosito T, Pires JA et al: A new extra-abdominal channel alternative to the Mitrofanoff principle: experimental and preliminary clinical experience. Int Braz J Urol 2009; 35: 205.

2. Liguori R, Rosito T, Pires JA et al: A new extra-abdominal channel alternative to the Mitrofanoff principle based on two lower ab- dominal cutaneous flaps: preliminary clinical experience. J Pediatr Urol, suppl, 2010; 6: S44.

3. Yachia D: A new continent vesicostomy technique: preliminary report. J Urol 1997; 157: 1633

Reply by Authors: We would like to thank Macedo for his interesting comment. We totally agree that the concept of a pedicled skin flap to create a conduit is not new, since skin flaps have been used in hypospadias surgery. That is why Daoud began using this type of conduit for continent cystostomy in 1999. The technique and our preliminary results were reported at the 2004 European Society for Paediatric Urology meeting in Regensburg. ${ }^{1}$

We are interested in the report of Rackley. This notion is, indeed, close to our technique, except we think that the proximal cutaneous incision could be avoided. In his comment Macedo presents the concept of 2 flaps, elevated from each side of the lower abdomen. One of our concerns regards the hairiness of the tube, which is particularly difficult to anticipate in young children. Macedo and Rackley do the cystostomy in the middle line, which can be hairy during adulthood. At the beginning of our experience we elected to use a paraumbilical pedicle to avoid the middle line.

As reported, we currently tend to use a lateral flap, which seems more convenient for that particular indication (figure 7 in article). The incision is then transverse and more discrete.

We have no experience with the technique of Yachia regarding the continence procedure, and we agree that this point of the technique can be difficult, especially in patients who have undergone multiple surgeries (eg previous bladder enlargement, cervical procedures). That is why we sometimes do the extramucosal incision laterally to avoid the post-scarring fibrosis. Our cutaneous flap is not systematized and we can then choose the most convenient place for the abdominal opening, according to previously stated principles and patient clinical status. As described by Cervellione in the editorial comment that accompanied our article, in our latest patients we pass the channel between the rectus sheath and the rectus muscle. We cannot yet assess the results, but maybe this easy to do gesture will shorten the extramucosal incision and limit the need for bladder exposure, at least in difficult cases. Dysfunctional bladder management still remains a challenge, and any technical gesture, new or already applied, must be thoroughly understood and discussed while keeping in mind the long-term outcomes.

1. Poli-Merol ML, Belouadah M, Challemet $C$ et al: Use of a cutaneous flap for continent vesicostomy: an interesting alternative. BJU Int, suppl, 2004; 93: 31

\title{
Re: Transplantation of Nonhematopoietic Adult Bone Marrow Stem/Progenitor Cells Isolated by p75 Nerve Growth Factor Receptor Into the Penis Rescues Erectile Function in a Rat Model of Cavernous Nerve Injury
}

\author{
M. Kendirci, L. Trost, B. Bakondi, M. J. Whitney, W. J. G. Hellstrom and J. L. Spees \\ J Urol 2010; 184: 1560-1566.
}

To the Editor: We wish to congratulate the authors on a beautifully conducted and written study. In an elegant fashion they show beneficial effects of transplantation of autologous rat multipotent 
stromal cells (rMSCs) and p75 positive selected rMSC on erectile function in a standardized rat model of bilateral cavernous nerve injury (CNI). Interestingly they propose paracrine signaling as the capital mechanism of action of MSC therapy for cavernous nerve injury based on a paucity of stem cell engraftment and a mesenchymal morphology of the rare stem cells remaining in the erectile tissue. Furthermore, they demonstrated a higher secretory capacity of rMSCs and p75 rMSCs compared to fibroblast controls.

A recent series of publications marks a shift in the paradigm that MSC effects are established by incorporation in the host tissue, toward a way of thinking that MSC might have important paracrine effects on the host tissue. Although in vitro (trans)differentiation is easily induced in MSCs, various studies outside the field of sexual medicine, have shown unsatisfactory results regarding in vivo engraftment of MSC. ${ }^{1}$ Furthermore, two important observations support the hypothesis that MSC have paracrine effects on the host tissue. The beneficial effects of MSCs have been observed within 3 days following transplantation, a time frame that is too small to allow for engraftment and differentiation. Also, and perhaps most striking, the beneficial effects of MSCs have been replicated using cell-free lysate and conditioned MSC culture medium. ${ }^{1,2}$ MSCs hold great secretory potential, which is confirmed in this study.

The authors importantly recognize that MSCs likely do not exert their beneficial effects by engraftment in the CNI rat model. Our group previously showed that mesenchymal stem cells cultured from adipose tissue (ADSC) were able to induce recovery of erectile function following CNI in rats. ${ }^{3}$ This recovery was accompanied by a recovery of neuronal nitric oxide synthase expression in the penile nerves, analogous with the proposal by Kendirci et al that MSC might work via potentiation of nerve regeneration. Interestingly these results were almost entirely replicated by injection of cell-free lysate derived from ADSC, thereby exposing the host to soluble molecules contained in ADSC while excluding the possibility of engraftment. Fall et al injected bone marrow mononuclear cells following CNI and showed retained PKH-26 cell label in the cavernous tissue surrounding the sinusoids but did not demonstrate individual cell morphology. ${ }^{4}$ Furthermore, no differentiation was observed. Fandel et al showed a time dependent decrease in penile ADSC numbers in CNI rats with almost none remaining at 28 days following intracavernous injection. ${ }^{5}$ Furthermore, ADSC conditioned medium has been shown to promote neurite outgrowth from cultured rat major pelvic ganglion. ${ }^{6}$ The results of these studies strongly argue for paracrine mechanisms of actions, while strong evidence for penile stem cell engraftment following CNI is generally lacking.

Additional research is indicated to characterize these findings further. Specifically following cancer treatment it is important to determine whether paracrine signaling molecules secreted by MSC could have tumorigenic effects.

Respectfully,

Maarten Albersen and Tom F. Lue
Department of Urology
University Hospitals Leuven
Leuven, Belgium
and Department of Urology
School of Medicine
University of California, San Francisco
San Francisco, California
e-mail: maarten.albersen@uzleuven.be

Reply by Authors: We appreciate the kind words of Albersen and Lue regarding our recent article. Their letter raises several important points regarding the mechanism(s) responsible for the benefits conferred by administering bone marrow MSCs (BMSCs), bone marrow mononuclear cells (BMCs), or ADSCs after tissue injury. ${ }^{2,3,7}$ Unlike the case with robust marrow engraftment of uncultured hematopoietic stem/progenitor cells after transplantation into ablated hosts, intravenous or intra-arterial infusion or direct injection of cultured adult nonhematopoietic stem/ progenitor cells into injured tissues frequently results in low long-term cell engraftment. Notably, despite low cell engraftment, adult stem/progenitor cell administration is often found to 
correlate with preservation or partial repair of tissue and, in many cases, significant functional improvement. ${ }^{1}$ These observations lend support to the "paracrine hypothesis", namely that rescue and repair of injured tissue after cell transplantation occurs primarily through the release of secreted growth factors, cytokines, and hormones by transplanted cells rather than through differentiation and direct replacement of injured cells.

Albersen et al recently began to address the paracrine hypothesis for ADSC treatment after cavernous nerve injury (CNI) by comparing the effects of administering ADSCs or ADSC lysates. Interestingly, they found little difference in the protection provided against erectile dysfunction by ADSCs or ADSC lysates. ${ }^{3}$ Although both treatments were effective, they may provide benefit through different mechanisms. Infusion of cell lysates may not reflect the composition of factors secreted by transplanted cells during short-term engraftment. Further study is now needed to determine whether administered adult stem/progenitor cells actually lyse in large numbers and release their cellular contents in an unregulated manner, or act transiently to secrete specific molecules in response to cues from injured tissues. Recent studies support the latter mechanism, although lysis of some cells and regulated secretion from others could conceivably occur simultaneously. ${ }^{4,5,6}$

We found that human BMSCs isolated from marrow aspirates by sorting against CD133 engrafted for 48 hours following direct injection into cortical peri-infarct areas after stroke. Human specific real-time polymerase chain reaction assays demonstrated that the CD133 BMSCs injected into peri-infarct areas increased their SDF-1 mRNA levels about 80-fold compared with CD133 BMSCs injected into sham operated cortical tissue. ${ }^{8}$ Lee et al reported that TSG-6, an anti-inflammatory protein, was produced and secreted by human BMSCs at significantly higher levels after the cells lodged in the lung following intravenous infusion. ${ }^{9}$ These results indicate that the expression and secretion of some reparative factors may be altered dramatically once cultured cells encounter different environments in vivo. Although typically thought to be beneficial, the ability of transplanted adult stem/progenitor cells to respond to new environments in vivo should be carefully considered, since such responses could vary for individuals with different case histories. Importantly this issue could contribute to variability in treatment outcomes.

An additional point raised by Albersen and Lue concerns the physiological aspects of benefit acquired through adult stem/progenitor cell transplantation after CNI. In addition to the biochemistry behind the effects, what cells are responding and how does their response lead to the prevention of erectile dysfunction after CNI? Direct neuroprotection is a possible mechanism, and this concept is in line with the observed recovery of neuronal nitric oxide synthase expression in penile neurons detected by Albersen et al after ADSC transplantation. ${ }^{3}$ The marked secretion by p75 MSCs of bFGF, a growth factor well known to provide neuroprotection, also supports the idea of direct neuroprotection in our CNI model. ${ }^{10}$ However, indirect physiological mechanisms are also possible. For example many of the growth factors secreted by non-hematopoietic progenitor cells are vasoprotective and/or angiogenic. Improved neuronal survival or function could occur indirectly because of improved vascular perfusion. Accordingly another avenue of future studies should focus on identifying physiological mechanisms in addition to biochemical mechanisms of treatment effects after cell transplantation.

Because cell engraftment may not actually be necessary to achieve the paracrine or secreted effects of adult stem/progenitor cells, it may be possible to infuse instead a cocktail of secreted molecules prepared from cultured cells or even defined combinations of proteins/peptides. In other studies with human CD133 BMSCs we found that intra-arterial infusion of serum-free concentrated conditioned medium (CD133 BMSC CdM) provided significantly greater preservation of cortical tissue after stroke compared with infusion of cells. ${ }^{11}$ Similarly CdM generated from human p75 selected MSCs may provide an effective treatment to protect against nerve damage after radical prostatectomy. Ultimately biochemical analysis of p75 MSC CdM or CdMs from other cells may lead to the identification of several active factors and a recipe for a powerful combination therapeutic to provide vasoprotection and/or neuroprotection after injury.

1. Crisostomo PR, Markel TA, Wang Y et al: Surgically relevant aspects of stem cell paracrine effects. Surgery 2008; 143: 577

2. Yeghiazarians $Y$, Zhang $Y$, Prasad $M$ et al: Injection of bone marrow cell extract into infarcted hearts results in functional improvement comparable to intact cell therapy. Mol Ther 2009; 17: 1250.
3. Albersen $M$, Fandel TM, Lin $G$ et al: Injections of adipose tissuederived stem cells and stem cell lysate improve recovery of erectile function in a rat model of cavernous nerve injury. J Sex Med 2010; Epub ahead of print. 
4. Fall PA, Izikki M, Tu L et al: Apoptosis and effects of intracavernous bone marrow cell injection in a rat model of postprostatectomy erectile dysfunction. Eur Urol 2009; 56: 716.

5. Fandel TM, Albersen M, Lin $\mathrm{G}$ et al: Tracking of injected adipose tissue-derived stem cells after cavernous nerve injury in rats: injuryinduced homing to the major pelvic ganglion. Unpublished data.

6. Bella AJ, Garcia M, Lin G et al: Adult adipose tissue derived stem cells enhance neurite outgrowth from the major pelvic ganglion of the rat. CUAJ 2007; 1: 200, abstract 2.01 .

7. Iso Y, Spees JL, Serrano $C$ et al: Multipotent human stromal cells improve cardiac function after myocardial infarction in mice without long-term engraftment. Biochem Biophys Res Commun 2007; 354: 700 .
8. Gnecchi M, Zhang Z, Ni A et al: Paracrine mechanisms in adult stem cell signaling and therapy. Circ Res 2008; 103: 1204.

9. Bakondi B, Shimada IS, Peterson BM et al: SDF-1 secreted by human CD133-derived MSCs promotes neural progenitor cell survival through CXCR7. Stem Cells Dev 2010; Epub ahead of print.

10. Lee RH, Pulin AA, Seo MJ et al: Intravenous hMSCs improve myocardial infarction in mice because cells embolized in lung are activated to secrete the anti-inflammatory protein TSG-6. Cell Stem Cell 2009; 5: 54.

11. Bakondi B, Shimada IS, Perry A et al: CD133 identifies a human bone marrow stem/progenitor cell sub-population with a repertoire of secreted factors that protect against stroke. Mol Ther 2009; 17: 1938.

\section{ERRATA}

\section{THIS MONTH IN PEDIATRIC UROLOGY}

Volume 185, Number 1, Page 3, Column 1: Four sentences before the last sentence in the commentary on "Late Phase Renal Scintigraphy and Ultrasound Screening of Urinary Tract Infection" should state: "Using the OR rule the sensitivity for detecting dilating VUR was $97 \%$ and the diagnostic odds ratio was 25.3. In this scenario of 533 patients only $336(63 \%)$ would undergo VCUG, resulting in failure to detect $9(6.2 \%)$ with dilating VUR. Using the "AND rule" the sensitivity and NPV decreased to $69 \%$ and $87 \%$, respectively, with a diagnostic odds ratio of 16.4, and only 177 (33.2\%) patients would undergo VCUG, resulting in failure to detect 39 (27\%) with dilating VUR."

\section{SHOCK WAVE LITHOTRIPSY AND HYPERTENSION RISK}

Volume 185, Number 1, Page 164: This study was also supported by the Rochester Epidemiology Project Grant number R01 AG034656 from the National Institute on Aging. 